\title{
Development and characterization of electric heating fabric based on silver coated nylon yarn
}

\begin{abstract}
Due to the recent development of wearable electronic textiles and smart textiles, the conductive fabrics and electrically heated garments have been extensively studied and applied in many fields. In this work an electric heating fabric was fabricated by sewing silver coated nylon yarn over the polyester fabric. In order to minimize the power consumption, instead of heating whole garment surfaces, local warming was preferred by stitching silver coated nylon yarn over polyester fabric in a specific pattern. The integration of silver coated nylon yarn and portable power supply used for warming function. The heating fabric properties such as power supply and saturation time for a particular temperature were investigated. The results showed that the increase in temperature above room temperature was $16^{\circ} \mathrm{C}$ and the power requirement for $1^{\circ} \mathrm{C}$ rise in temperature was 0.92 Watts.
\end{abstract}

Volume I Issue 6 - 2017

Thilagavathi G,'Muthukumar N,' Kannaian $\mathrm{T}^{2}$ 'Department of Textile Technology, PSG College of Technology, India

${ }^{2}$ Department of Electronics, PSG College of Arts \& Science, India

Correspondence: Thilagavathi G, Department of Textile Technology, PSG College of Technology, Coimbatore-641004, India,Tel 9894769906, Email thilagapsg@gmail.com

Received: March 29, 2017| Published: May II, 2017

Keywords: electro textiles, heating fabric, power supply, silver coated nylon yarn, temperature

Abbreviations: $\mathrm{C}$, celsius; D, denier; f, filament; V, voltage; GSM, grams per square meter; $\mathrm{cm}$, centimeter; AATCC, american association of textile chemists and colourists

\section{Introduction}

Electro-textiles can be defined as textiles with unobtrusively built-in electronic and photonic functions. They are mostly used for electromagnetic shielding, anti static and heating purposes, and also for soft circuits: electric circuits or sensors made out of a combination of special fabrics, threads, yarns and electronic components. Electrical functions can be embedded in textiles by using weaving, knitting and embroidery or nonwoven production techniques. The integration of electronic properties directly into the clothing environment carries some advantages such as increased comfort, mobility, usability and aesthetic properties. However, there are some challenges to be addressed. Yarns that are used for making cloth should be fine and elastic in order to ensure the wearer's comfort. The fibers have to be able to withstand handling and fabrics should have low mechanical resistance to bending and shearing which means they can be easily deformed and draped. ${ }^{1,2}$

In recent years, lot of research going on in the field of the conductive fabrics and electrically heating garments. Heating garments are developed especially for use by people who work outside during their day, such as military applications, security services, and other civil services. It is also used in medical fields such as electrotherapy treatment and medical blanket for maintaining patient's body temperature. By the integration of electro-conductive properties in the fabric structure, a flexible textile can be obtained that does not reduce the quality of comfort, maintenance and wearability of clothing. Generally, electrical heating products use embedded heating elements to generate heat. Commonly steel based conductive wires are used to heat the fabric for heat control. Carbon fiber heating elements are also popular in electric heating garments. The carbon fiber heating element has good heat efficiency and can generate heat uniformly and rapidly. A chemical heating pad uses a reaction of chemical substances to generate heat e.g. chemical energy can be turned into heat energy by oxidization the reagents are kept in separate compartments within the pad. When the user squeezes the pad, a barrier ruptures and the reagents mix, producing heat. Wang et al summarized advantages and disadvantages of several kinds of personal heating garments. ${ }^{3}$

Ozan et al. ${ }^{4}$ developed heating garment by using steel-based conductive yarns with portable power supplies and controlling electric circuit and evaluated the performances of the heating garments on a thermal mannequin in cold weather environments. In another study the authors showed that performances of the heating fabric were closely correlative with power supply and current amount. ${ }^{5}$ Hao et al. ${ }^{6}$ developed plain woven flexible heating fabrics by weaving intermittently silver filaments and showed that strong positive linear correlation between rated power and utmost ascending temperature of the fabric and between power consumption and presetting equilibrium temperature of heating fabric. Tong $\mathrm{J}$ et al. ${ }^{7}$ studied the electrical resistance of knitted fabrics embedded with conducting yarns at different temperatures and shown that the resistance of the knitted conductive fabric decreases significantly when its temperature is rising. In this work, we have developed electrical heating fabric by sewing silver coated nylon yarn over the polyester fabric. Then the relation between the power supply and temperature of the developed fabric was investigated.

\section{Materials and methods}

\section{Materials used}

The heating fabric was fabricated by stitching conductive yarn over polyester fabric. $100 \%$ polyester plain woven fabric made of warp $75 \mathrm{D}$ and weft $150 \mathrm{D}$ (GSM 86) was used as base fabric for the development of heating fabric. Polyester fabric acts as an insulator, endures abrasion very well and dries fast. The selection of conductive filament is more crucial as it is the main component of heating fabric. Silver coated nylon yarn of $117 \mathrm{D} / 17 \mathrm{f}$ was purchased for the development of heating fabric. These yarns are highly conductive, more flexible, soft and pliable. 


\section{Design of heating fabric}

For development of electrically heating fabric, the silver coated nylon yarn was stitched over the polyester base fabric in parallel combination as shown in Figure 1 to provide parallel power supply. In parallel connection the resultant resistance of the design is less and it can produce maximum heating effects. In order to make connection with power supply, an additional stitch was given in serial manner.

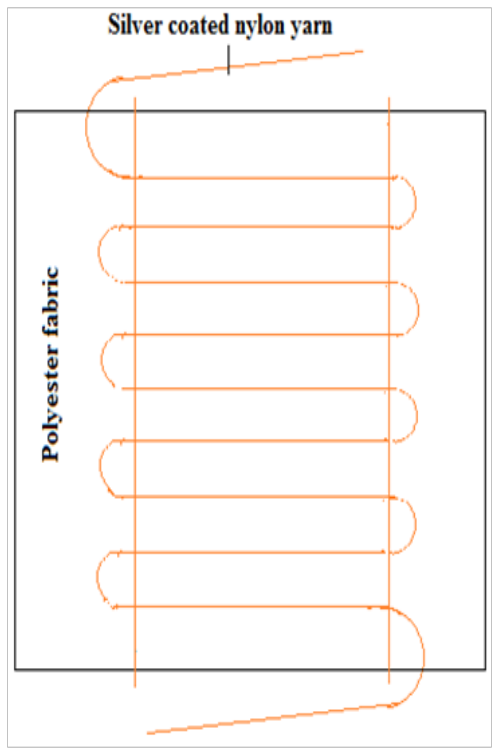

Figure I Design of Electric Heating Fabric.

\section{Characterization methods}

Measurement of electrical resistance of silver coated nylon yarn: Electrical resistance measurements were performed on the silver coated nylon yarn after conditioning the samples in a standard atmosphere. The resistance was measured ten times and the average value was taken. The American Association of Textile Chemists and Colourists (AATCC) test method 76-1995 was used to measure the resistance of the conductive yarn.

Measurement of heat generating property: The heat generating property of the developed fabric was investigated by monitoring the temperature of fabric surface upon applying an electrical current, where a commercial $9 \mathrm{~V}$ battery was used as power supply. The temperature of fabric surface was measured using a thermo couple mounted on the surface of the fabric under the atmospheric condition. The experimental set up used in this study shown in the Figure 2.

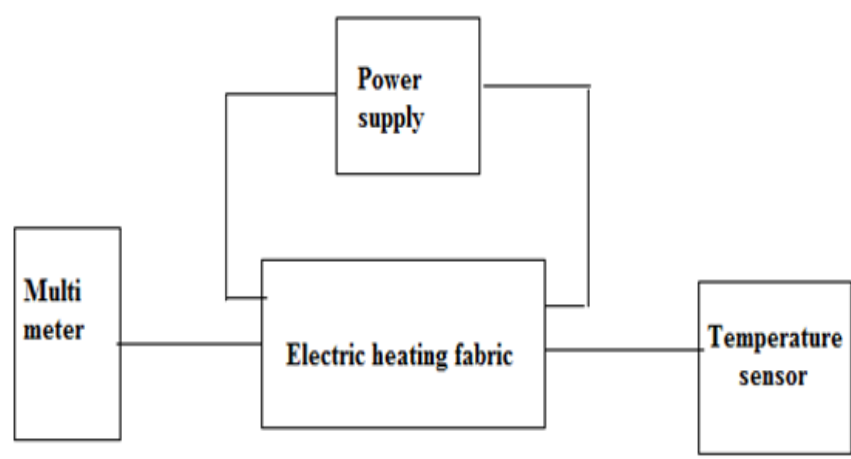

Figure 2 Experimental set up for heating fabric characterization.

\section{Results and discussion}

\section{Thermo-electric properties of heating fabric}

The electrical resistance of the silver-coated multifilament nylon yarn of $117 \mathrm{D} / 17 \mathrm{f}$ was measured and the results are shown in Figure 3 . The test was carried out between the lengths of $1 \mathrm{~cm}$ to $10 \mathrm{~cm}$. The change in resistance was found to be directly proportional to the length. For studying the relation between power applied and temperature, the temperature of the developed fabric was raised above the ambient room temperature using portable power supply. This temperature rise is not the average temperature rise of the whole fabric. So in order to find the average temperature rise of fabric, it was decided to keep the heating fabric inside a cartoon box. Fifteen trials were taken, and the corresponding temperature rise, power applied and saturation time have been recorded. The average temperature rise of fabric with respect to power applied and time was calculated and shown in Figure 4. The calculated t-value (1.83) was smaller than t-table value at $99 \%$ confidence level (2.977) indicating that there is no significant difference between the trials.

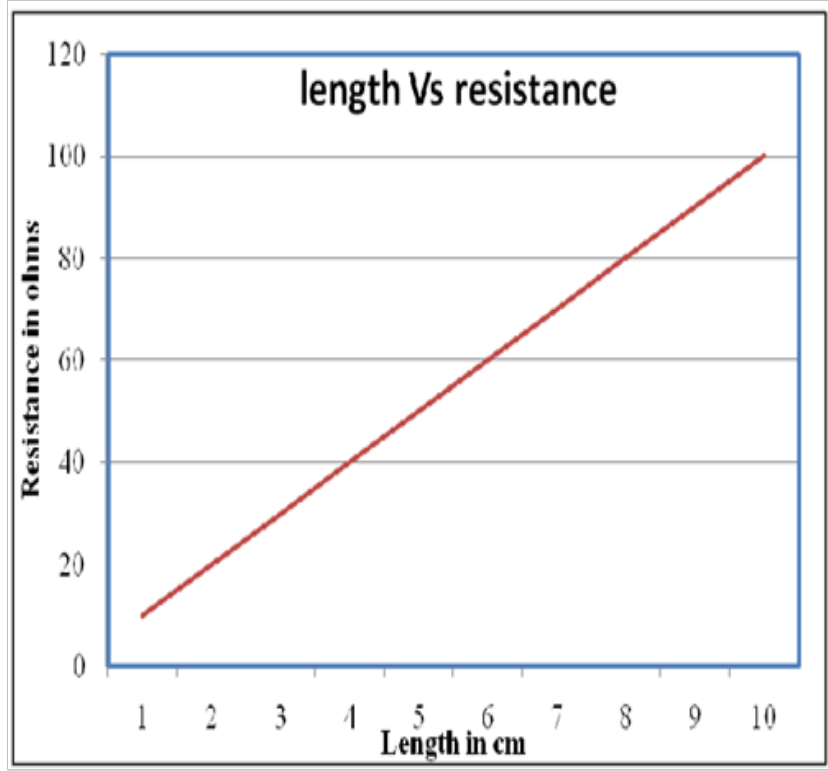

Figure 3 Effect of yarn length on resistance value.

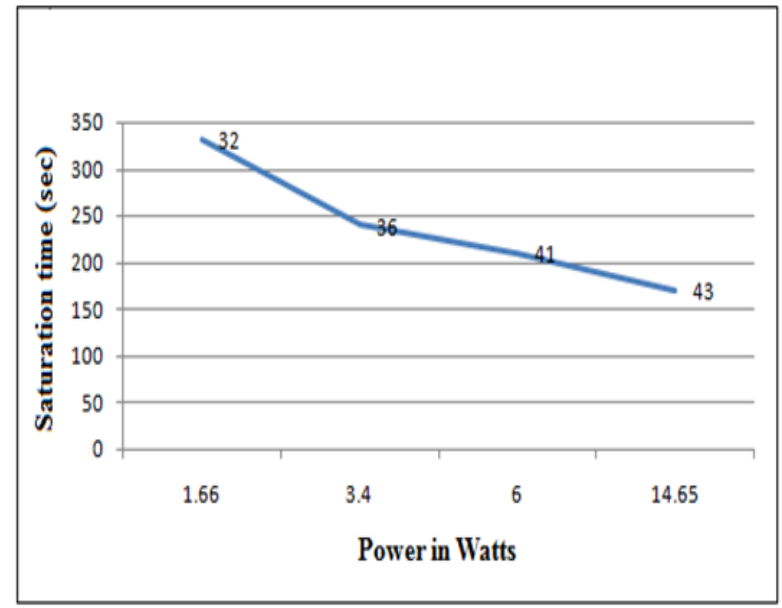

Figure 4 Relation between power supplied, temperature and saturation time. 
It was observed that the temperature of the developed fabric rises during the power application. When power is applied to the conductive fabric heat generated in the conducting yarns raises the temperature of the fabric. It is known that an increase in temperature will lead to a higher resistivity of silver. This is in fact opposite to the actual decrease in the fabric resistance. The fabric takes more than 5minutes to raise the temperature from $27^{\circ} \mathrm{C}$ to $32^{\circ} \mathrm{C}$ for 1.66 Watts power. The temperature of the developed fabric rises quickly from $27^{\circ} \mathrm{C}$ to $43^{\circ} \mathrm{C}$ within 3-4minutes when 14.65 watts power is applied. It was also observed that the behavior of the developed heating fabric remained the same for many number of repeated heating and cooling cycles.

Figure 5 shows the recorded temperature as a function of the applied electric power. The temperature of fabric rises quickly during initial power supply i.e up to 6 Watts and then experiences a continuous and slow increase during the rest of the heat time. It can also be observed from Figure 5 that the co-efficient of determination $\left(\mathrm{R}^{2}\right)$ of the linear regression curve is 0.975 . This indicates that there is a good linear relationship between the temperature rise and power applied to the fabric. From the slope of the trend line, the amount of power required to rise one degree temperature of the developed heating fabric is found to be $0.92^{\circ} \mathrm{C} /$ Watt. The heat generating period can be increased much longer when the temperature is controlled at a desired level such as $40^{\circ} \mathrm{C}$ by applying a temperature controller.

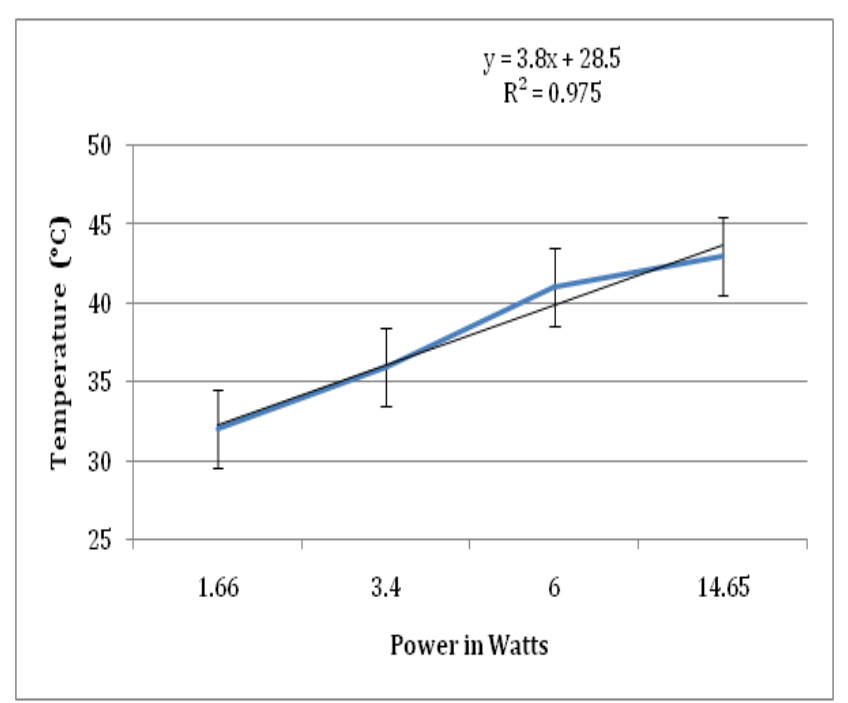

Figure 5 Relation between power supplied and temperature

The heating fabric developed in this study may have wide range of applications including the flexible mobile surface-heating element for medical or other uses. The developed heating fabrics are highly flexible and can be made wearable. Further, the fabric is breathable and soft so that it will not be uncomfortable. It can be sewn into the dress at those points where warming of the body part is essential. For example, during winter, the fingers, toes and palm can be warmed up slightly to make the person comfortable. The switch for on/off can be integrated within the person's jacket so that he can economize the power as per the needs.

\section{Conclusion}

From this study, it has been concluded that the electrical heating fabric can be developed by sewing silver coated nylon yarn over polyester fabric. It has been shown that the heating fabric with an area of $18 \times 10 \mathrm{~cm}$ can be heated up to approximately $12^{\circ} \mathrm{C}$ above ambient temperature with limited amount of power supply around 6 Watts. The relationship between the temperature of a fabric and the power applied is analyzed and this finding can be used for practical heated temperature prediction. Being flexible and breathable, such fabrics can be used for making heating pads and can be integrated into apparel.

The developed electric heating fabric can be an alternative for thermal insulation shawls to protect the humans from extreme cold weather and also used in medical field for localized heating of affected areas. It can be used for arthritis patients for heating knee and elbow. It can be used for the people who work outside their day under cold weather, such as military applications, security services and other civil services.

\section{Acknowledgements}

The authors thank M/s PSG TECHS COE INDUTECH, Coimbatore for carrying out this research.

\section{Conflict of interest}

Author declares there is no conflict of interest in publishing the article.

\section{References}

1. Rossi D, Carpi F, Lorussi F. Electro active fabrics and wearable bio monitoring devices. AUTEX Res J. 2003;3(4):180-185.

2. Van Langehove L. Smart textiles for medicine and healthcare: materials, systems and applications. CRC Press, New York; 2007. p. 90.

3. Wang F, Gao C, Kuklane K, et al. A review of technology of personal heating garments. Int J Occup Saf Ergon. 2010;16(3):387-404.

4. Ozan K, Ender B, Ozge S. Implementation of steel-based fabric panels in a heated garment design. Text Res J. 2009;79(16):1427-1437.

5. Ozan K and Ender B. Heating behaviors of metallic textile structures. Int J Cloth Sci Technol. 2009;21(2/3):127-136.

6. Hao L, Yi Z, Li C, et al. Development of flexible heating fabric based on conductive filaments. Measurement. 2012;45(7):1855-1865.

7. Tong J, Ding F, Tao X, et al. Temperature effect on the conductivity of knitted fabrics embedded with conducting yarns. Text Res J. 2014;84(17):1849-1857. 